\title{
Article \\ Landscape Perception Identification and Classification Based on Electroencephalogram (EEG) Features
}

\author{
Yuting Wang ${ }^{1,2}$, Shujian Wang ${ }^{1,2}$ and Ming $\mathrm{Xu}^{1,2,3, *}$ \\ 1 Henan Key Laboratory of Earth System Observation and Modeling, Henan University, Kaifeng 475004, China; \\ wyt2019@vip.henu.edu.cn (Y.W.); wangsj@henu.edu.cn (S.W.) \\ 2 College of Geography and Environmental Science, Henan University, Kaifeng 475004, China \\ 3 BNU-HKUST Laboratory for Green Innovation, Advanced Institute of Natural Sciences, \\ Beijing Normal University at Zhuhai, Zhuhai 519087, China \\ * Correspondence: mingxu@henu.edu.cn
}

check for

updates

Citation: Wang, Y.; Wang, S.; Xu, M. Landscape Perception Identification and Classification Based on

Electroencephalogram (EEG)

Features. Int. J. Environ. Res. Public

Health 2022, 19, 629. https: / /doi.org/

$10.3390 /$ ijerph19020629

Academic Editor: Paul B

Tchounwou

Received: 24 November 2021

Accepted: 28 December 2021

Published: 6 January 2022

Publisher's Note: MDPI stays neutral with regard to jurisdictional claims in published maps and institutional affiliations.

Copyright: (C) 2022 by the authors. Licensee MDPI, Basel, Switzerland. This article is an open access article distributed under the terms and conditions of the Creative Commons Attribution (CC BY) license (https:// creativecommons.org/licenses/by/ $4.0 /)$.

\begin{abstract}
This paper puts forward a new method of landscape recognition and evaluation by using aerial video and EEG technology. In this study, seven typical landscape types (forest, wetland, grassland, desert, water, farmland, and city) were selected. Different electroencephalogram (EEG) signals were generated through different inner experiences and feelings felt by people watching video stimuli of the different landscape types. The electroencephalogram (EEG) features were extracted to obtain the mean amplitude spectrum (MAS), power spectrum density (PSD), differential entropy (DE), differential asymmetry (DASM), rational asymmetry (RASM), and differential caudality (DCAU) in the five frequency bands of delta, theta, alpha, beta, and gamma. According to electroencephalogram (EEG) features, four classifiers including the back propagation (BP) neural network, k-nearest neighbor classification (KNN), random forest (RF), and support vector machine (SVM) were used to classify the landscape types. The results showed that the support vector machine (SVM) classifier and the random forest (RF) classifier had the highest accuracy of landscape recognition, which reached $98.24 \%$ and $96.72 \%$, respectively. Among the six classification features selected, the classification accuracy of MAS, PSD, and DE with frequency domain features were higher than those of the spatial domain features of DASM, RASM and DCAU. In different wave bands, the average classification accuracy of all subjects was $98.24 \%$ in the gamma band, $94.62 \%$ in the beta band, and $97.29 \%$ in the total band. This study identifies and classifies landscape perception based on multi-channel EEG signals, which provides a new idea and method for the quantification of human perception.
\end{abstract}

Keywords: unmanned aerial vehicle (UAV); electroencephalogram (EEG) features; landscape perception; machine learning

\section{Introduction}

Traditionally, landscape classification has always been concerned with descriptive analysis involving the physical characteristics of a landscape based on basic surveys and specifications. However, the combination of the subjective, visual appreciation of scenery and the more objectively describable physical elements seems to have been strong resistance to the idea of landscape classification [1,2]. Yet there seems to be no classification problems if we restrict ourselves to the more limited physical (namely landform and land-use) concept of landscape. Recent studies have evaluated the consistency of the existing manually constructed natural landscape classification with a machine learning-based approach in order to explain the variable importance of the differentiation between natural landscape types [3]. Landscape classification of Central Europe was based on the cluster analysis of principal components, which would be used for further assessment of ecosystem services within the focus region [4]. The later $3 S$ technology also provides a strong driving force for landscape classification research. The idea of using GEOBIA and a supervised classifier to 
classify ground features and extract landscape information from UAV images has been recognized by most researchers [5]. At present, multi temporal remote sensing images, which have been widely applied, can effectively improve the accuracy of feature information extraction because they can provide spectral information on different time phases [6-8]. In the field of remote sensing, various depth learning technologies and high-resolution images have made indelible contributions to landscape classification. However, these are classified based on the objective feature attributes of the landscape itself, and there are relatively few studies on objective measurement and recognition based on landscape perception.

The traditional research methods of landscape perception mainly focus on scenic beauty evaluation (SBE) [9-11], the semantic differential method (SD) [12,13], and the analytic hierarchy process (AHP) [14]. The landscape is displayed through photos or field observation. For example, the aesthetic appearance of photographic images are used as a monitoring tool for coral reefs [15]. In addition, subjective perception judgments have been used on different types of pictures to quantify 47 sites along the Colorado River in the Grand Canyon National Park [16]. Qualitative and semi-quantitative research is conducted on the landscape by the fuzzy evaluation method of landscape description and overall perception [17]. However, from the perspective of psychology, Smith believes that the color of the plant itself can help us relieve stress [18]. The human eyes are particularly sensitive to green. The body will automatically reduce excitement when it sees green. At the same time, the "savanna hypothesis", the "refuge theory" [19], and the "biological theory" [20] all show that there is a genetic basis for the human preference for landscape. Evolutionary psychology also shows that people's pro naturalness is associated with basic survival needs, such as seeking refuge [15,21,22]. From the perspective of human perception, these notions suggest that people will produce different physiological responses in different environments or landscapes, which are related to the unique pattern of brain activity. Therefore, based on physiological electroencephalography (EEG), we can provide a new view of landscape analysis. The above shows that people will have different physiological reactions when they see different landscapes or when they are in different environments.

The measurement of brain activity is an objective way of assessing the physiological perception of engagement with the landscape, environment, or other objects [23-25]. Brain imaging is helpful to measure the effects of unconscious stimuli [26,27]. EEG frequency features have commonly been used in EEG signals. Generally, the frequency range of high amplitude signals observed is different when subjects are in calm state and when they are in a stimulated state [28,29]. EEG features (frequency domain features, time domain features, and spatial domain features) in EEG signals represent the brain region activities. With the continuous development of neuroscience and the trend of interdisciplinarity, thus far, there have been many studies using EEG technology and machine learning for recognition with good classification accuracy, e.g., emotion recognition [30-33], object structure recognition [34,35], color recognition [36], landscape and animal image recognition [37]. Some studies have gradually applied EEG technology to different fields, including environmental perception and landscape assessment [38-41], while others have also explored the impact of specific environmental characteristics on the natural environment [42-44]. Therefore, this study combines the experimental technology of neurology and UAV aerial video to identify and classify the landscape types from the perspective of human perception and compares the advantages and disadvantages of different classification features and classification methods.

\section{Materials and Methods}

\subsection{Materials}

Five landscape videos were selected for each landscape type. Seven professors with more than five years of experience in landscape design were asked to rate each landscape video on a scale of 1-10. The highest-scoring landscape videos were chosen to become the experimental materials according to the cumulative scores. The stimuli included seven highdefinition videos, mainly including different landscape types (forest, wetland, grassland, 
desert, water, farmland, and city) (Figure 1). The subjects were stimulated through video experience. During the experiment, conductive paste was used to reduce scalp resistance below $5 \mathrm{~K} \Omega$. In the laboratory, the room was completely closed and without noise. A 15-inch display device was put in front of the subjects. There were seven different videos, each of which remained on screen for about $40 \mathrm{~s}$ ( 2 repeats for each stimuli video). After a video was displayed, the volunteers had a minute to rest, mainly to calm their emotions and to not affect the next video. Then the next landscape video was played, followed by a rest, and so on until the end of the whole experiment. The whole process lasted for about $25 \mathrm{~min}$. The sequence in which the videos played during the experiment is random.

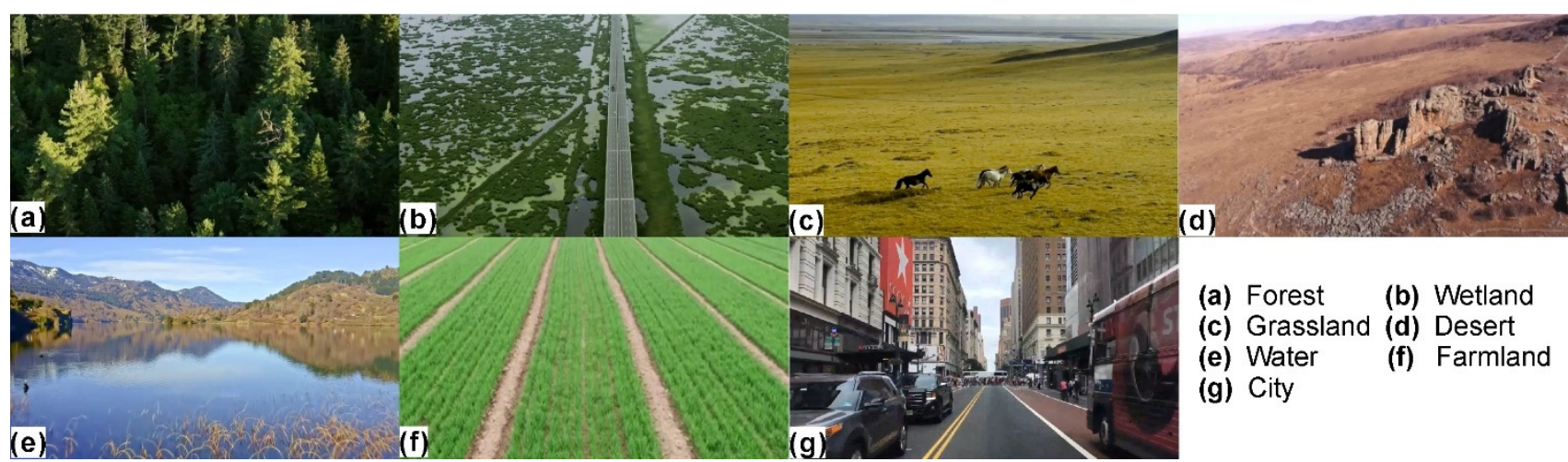

Figure 1. Experimental video materials.

\subsection{Subjects}

A total of 20 volunteers aged between 25 and 55, 12 women and 8 men, participated in the experimental procedure. The participants had varied employment situations, such as university staff, social workers of various industries, and university graduates. We described the purpose of the experiment before beginning, and participants were asked to be right-handed, with no color blindness, and in good physical health, without any history of a mental disorder. Additionally, a video was played in the lab, during which the participants were asked to stay as still as possible so that interferences such as EMG did not increase. If they met the requirements and agreed to continue the experiment, they then signed an informed consent form before testing. The study was approved by the school's ethics committee.

\subsection{Method}

In this study, the Active System produced by Brain Products (LiveAmp) with 32 channels was used to obtain the signals of the brain activity (Figure 2). The experiment used the international 10-20 system and a 32-channel electrode cap. The video stimuli were displayed in random order, and each landscape type was repeated twice for a total of 14 short videos. The original EEG data were analyzed by the EEGlab, which is a toolbox for processing continuous EEG signals.

\subsection{Statistical Analysis}

In this study, frequency domain features and spatial domain features are selected as important indicators of landscape recognition. The frequency domain features include: mean amplitude spectrum (MAS) [45], power spectrum density (PSD), and Differential Entropy (DE) [33,46,47], and spatial domain features include: differential asymmetry (DASM) and rational asymmetry (RASM) [48], and differential caudality (DCAU) [33]. 


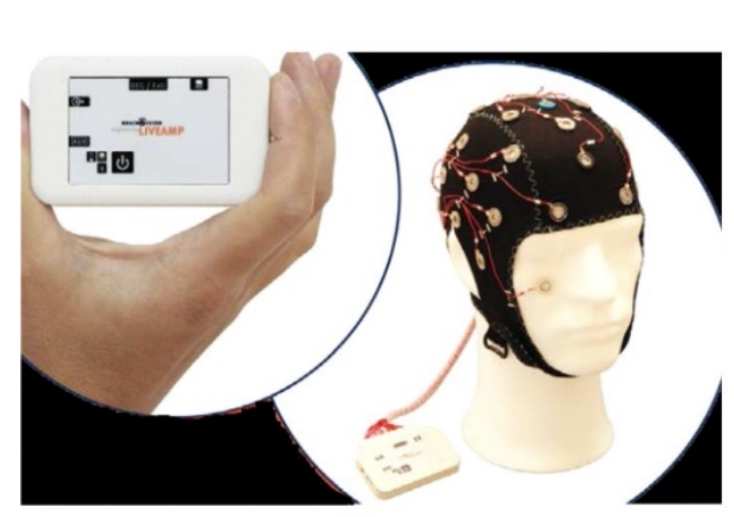

(a)

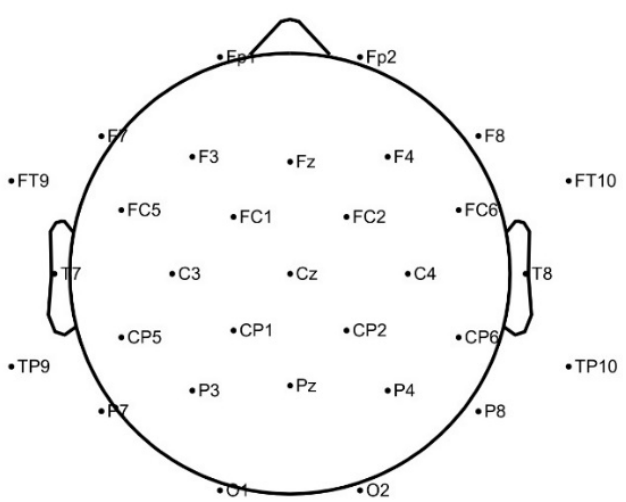

(b)

Figure 2. Brain Products (LiveAmp) in the experiment. (a) Brain Products (LiveAmp) with 32 channels; (b) The electrode position of international 10-20 system with 32 channels.

The EEG signals were detrended using the average of left and right mastoids as a reference. Then, $250 \mathrm{~Hz}$ downsampling and $0.5-70 \mathrm{~Hz}$ filtering were performed to obtain the preprocessed EEG datasets. Third, the eye electrical, electromyography, electrocardiography, power frequency interference, and other disturbance artefacts were removed by independent component analysis (ICA) [49-52]. Next, the EEG signals were segmented into contiguous $2 \mathrm{~s}$ windows, and any segments which retained artefacts were rejected $[53,54]$. Therefore, a total of $5600(7 \times 2 \times 20 \times 20)$ data samples were generated. In other words, there were a total of 7 landscape categories, and each video stimulus was repeated twice. Each subject could get 20 segments for $2 \mathrm{~s}$ of each video stimulus, with a total of $20 \mathrm{sub}$ jects. Then, Fast Fourier transform was used to extract frequency band information. The frequency-domain features were extracted to obtain the logarithmic frequency energy values of the waves in five frequency bands: delta (1-4 Hz), theta (4-8 Hz), alpha (8-13 Hz), beta $(13-30 \mathrm{~Hz})$, and gamma $(30-70 \mathrm{~Hz})$ [55]. The sample size of each subject in each frequency band is 280 characteristic data. Each frequency band contains the data of 29 electrode channels, and the total frequency band contains 145 electrode channels, that is, the samples of 5 frequency bands have a structure of $29 \times 280$, the sample structure of the total frequency band is $145 \times 280$ (Table 1$)$.

Table 1. Dimensions of each EEG feature.

\begin{tabular}{ccccccc}
\hline Feature & Delta & Theta & Alpha & Beta & Gamma & Total \\
\hline MAS & 29 & 29 & 29 & 29 & 29 & 145 \\
PSD & 29 & 29 & 29 & 29 & 29 & 145 \\
DE & 29 & 29 & 29 & 29 & 29 & 145 \\
DASM & 13 & 13 & 13 & 13 & 13 & 65 \\
RASM & 13 & 13 & 13 & 13 & 13 & 65 \\
DCAU & 11 & 11 & 11 & 11 & 11 & 55 \\
\hline
\end{tabular}

Firstly, four classifiers including the back propagation (BP) neural network algorithm (BP), k-nearest neighbor classification algorithm (KNN), random forest algorithm (RF), and support vector machine algorithm (SVM) were used to classify different landscape types of brain waves after the pretreatment of the EEG signals. Then, the recognition accuracy in different situations was obtained, and the accuracy of different classification methods for landscape type recognition was compared, in order to reflect the differences in people's perceptions of different landscape types.

The EEG data were randomly divided into training (80\%) and test (20\%) data. For our purposes, we have used 10-fold cross-validation to train and test extracted features for all classifiers. For KNN, we used k = 5 for a baseline in comparison with other classifiers. For random forest, we constructed the tree number with 500. We used LIBSVM software [56] to 
implement the SVM classifier and employ linear kernel, the back propagation (BP) neural network algorithm was also used.

Statistical analysis and data processing were mainly completed using MATLAB and R language. Figure 3 displays the experimental process.

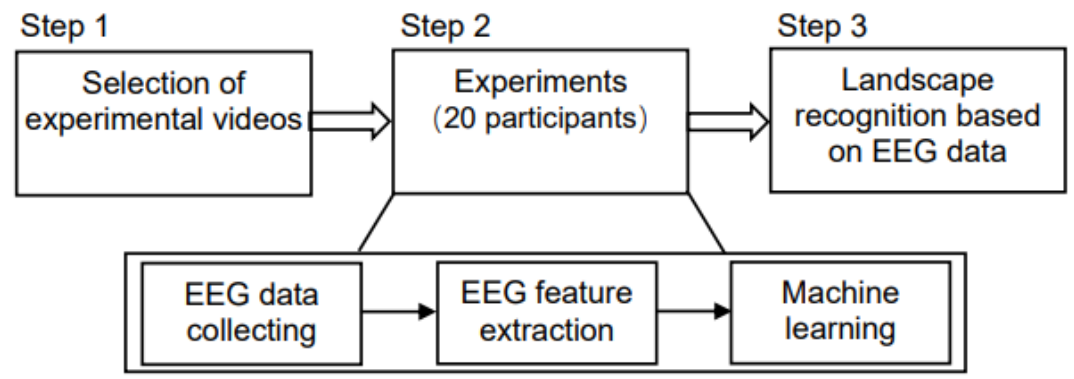

Figure 3. Flow chart of the experiment.

\section{Results}

\subsection{The Classification Effect of Different Classifiers}

The classification accuracy of the four classifiers for different landscape types are displayed in Table 2 and Figure 4. For all EEG features, the SVM classifier and RF classifier had the highest landscape recognition accuracy, followed by KNN, and BP had the lowest classification accuracy among the four classifiers. Meanwhile, Figure 5 shows the highest classification accuracy of different classifiers in 20 groups of subjects in all bands of brain waves, and also shows that the classification accuracy of SVM and RF was higher than that of KNN and BP.

Table 2. The accuracy of landscape perception and recognition based on different EEG features and different classifiers.

\begin{tabular}{|c|c|c|c|c|c|c|c|}
\hline Feature & Classifier & Delta (\%) & Theta (\%) & Alpha (\%) & Beta (\%) & Gamma (\%) & Total (\%) \\
\hline \multirow{4}{*}{ MAS } & $\mathrm{BP}$ & $20.38 \pm 5.74$ & $19.08 \pm 5.82$ & $22.67 \pm 7.18$ & $51.45 \pm 11.49$ & $69.2 \pm 11.15$ & $48.69 \pm 10.25$ \\
\hline & KNN & $40.07 \pm 11.85$ & $33.39 \pm 11.2$ & $44.79 \pm 15.1$ & $83.91 \pm 11.98$ & $95.28 \pm 4.17$ & $69.54 \pm 18.81$ \\
\hline & RF & $47.13 \pm 10.54$ & $40.27 \pm 12.56$ & $52.63 \pm 14.69$ & $90.33 \pm 6.77$ & $96.36 \pm 3.32$ & $96.51 \pm 3.33$ \\
\hline & SVM & $53.02 \pm 11.51$ & $45.96 \pm 12.74$ & $62.62 \pm 15.82$ & $94.62 \pm 4.95$ & $97.63 \pm 2.82$ & $96.13 \pm 4.38$ \\
\hline \multirow{4}{*}{ PSD } & $\mathrm{BP}$ & $19.08 \pm 5.41$ & $21.09 \pm 6.09$ & $24.55 \pm 8.11$ & $52.01 \pm 12.16$ & $69.36 \pm 11.39$ & $47.12 \pm 12.28$ \\
\hline & KNN & $40.21 \pm 10.63$ & $42.1 \pm 12.61$ & $48.63 \pm 17.1$ & $81.28 \pm 14.66$ & $94.13 \pm 5.48$ & $60.47 \pm 18.52$ \\
\hline & RF & $48.02 \pm 11.85$ & $51.07 \pm 14.58$ & $57.64 \pm 16.24$ & $88.84 \pm 8.12$ & $96.72 \pm 3.10$ & $96.52 \pm 3.34$ \\
\hline & SVM & $49.47 \pm 8.95$ & $58.23 \pm 12.38$ & $67.62 \pm 14.36$ & $92.55 \pm 6.63$ & $96.90 \pm 3.13$ & $93.98 \pm 6.79$ \\
\hline \multirow{4}{*}{$\mathrm{DE}$} & $\mathrm{BP}$ & $19.86 \pm 5.93$ & $21.84 \pm 6.79$ & $24.14 \pm 8.27$ & $51.41 \pm 12.22$ & $69.65 \pm 11.51$ & $50.71 \pm 9.21$ \\
\hline & KNN & $39.36 \pm 11.32$ & $41.29 \pm 15.24$ & $49.2 \pm 17.41$ & $83.13 \pm 12.83$ & $94.62 \pm 4.71$ & $86.14 \pm 11.32$ \\
\hline & $\mathrm{RF}$ & $48.21 \pm 10.84$ & $50.71 \pm 13.91$ & $57.73 \pm 15.94$ & $89.03 \pm 8.67$ & $96.51 \pm 3.15$ & $96.61 \pm 3.33$ \\
\hline & SVM & $55.56 \pm 9.72$ & $60.17 \pm 14.13$ & $69.04 \pm 14.76$ & $94.83 \pm 4.7$ & $98.24 \pm 2.31$ & $97.29 \pm 3.09$ \\
\hline \multirow{4}{*}{ DASM } & $\mathrm{BP}$ & $18.41 \pm 4.83$ & $19.39 \pm 6.12$ & $22.24 \pm 7.46$ & $38.82 \pm 11.99$ & $56.13 \pm 13.26$ & $37.74 \pm 10.63$ \\
\hline & KNN & $33.09 \pm 8.71$ & $33.64 \pm 13.03$ & $40.80 \pm 18.19$ & $75.36 \pm 14.15$ & $89.93 \pm 8.53$ & $81.32 \pm 11.64$ \\
\hline & RF & $42.57 \pm 8.2$ & $40.87 \pm 12.86$ & $47.01 \pm 16.3$ & $80.59 \pm 9.46$ & $92.08 \pm 5.86$ & $93.45 \pm 5.52$ \\
\hline & SVM & $42.44 \pm 8.12$ & $45.82 \pm 12.67$ & $55.14 \pm 15.99$ & $83.88 \pm 9.49$ & $93.26 \pm 6.29$ & $92.08 \pm 7.04$ \\
\hline \multirow{4}{*}{ RASM } & $\mathrm{BP}$ & $14.83 \pm 3.78$ & $14.54 \pm 3.29$ & $14.54 \pm 3.87$ & $24.93 \pm 10.84$ & $41.38 \pm 14.35$ & $26.71 \pm 10.20$ \\
\hline & KNN & $24.23 \pm 6.90$ & $23.78 \pm 8.03$ & $27.62 \pm 10.12$ & $70.59 \pm 13.21$ & $85.46 \pm 8.79$ & $41.49 \pm 13.01$ \\
\hline & RF & $37.99 \pm 8.42$ & $34.66 \pm 10.43$ & $41.42 \pm 14.73$ & $80.02 \pm 11.95$ & $91.35 \pm 6.59$ & $93.49 \pm 5.70$ \\
\hline & SVM & $26.42 \pm 6.63$ & $20.43 \pm 6.72$ & $23.19 \pm 10.37$ & $65.88 \pm 14.20$ & $96.52 \pm 3.35$ & $75.27 \pm 12.52$ \\
\hline \multirow{4}{*}{ DCAU } & $\mathrm{BP}$ & $14.22 \pm 2.72$ & $14.88 \pm 3.16$ & $14.54 \pm 2.99$ & $24.23 \pm 9.06$ & $39.96 \pm 13.42$ & $26.37 \pm 10.53$ \\
\hline & KNN & $25.25 \pm 9.00$ & $25.13 \pm 8.56$ & $27.39 \pm 8.04$ & $54.62 \pm 16.93$ & $80.01 \pm 8.11$ & $38.36 \pm 18.20$ \\
\hline & $\mathrm{RF}$ & $31.81 \pm 11.14$ & $33.64 \pm 11.57$ & $38.60 \pm 10.90$ & $71.76 \pm 13.07$ & $85.86 \pm 9.42$ & $86.16 \pm 10.63$ \\
\hline & SVM & $20.71 \pm 6.28$ & $20.81 \pm 7.49$ & $19.96 \pm 6.81$ & $66.71 \pm 10.82$ & $87.46 \pm 7.29$ & $79.09 \pm 8.97$ \\
\hline
\end{tabular}




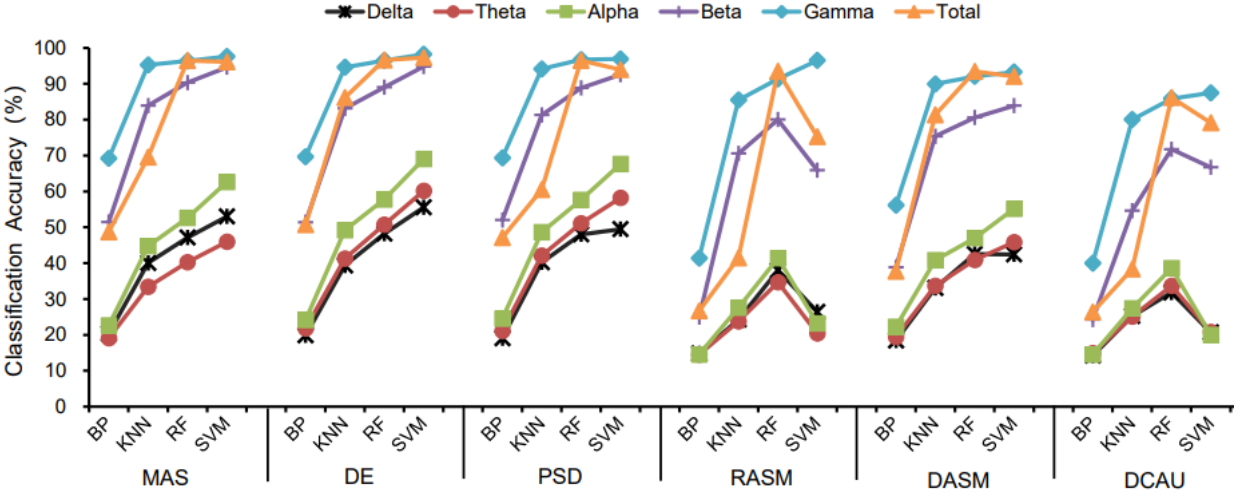

Figure 4. The classification results of different classifiers.

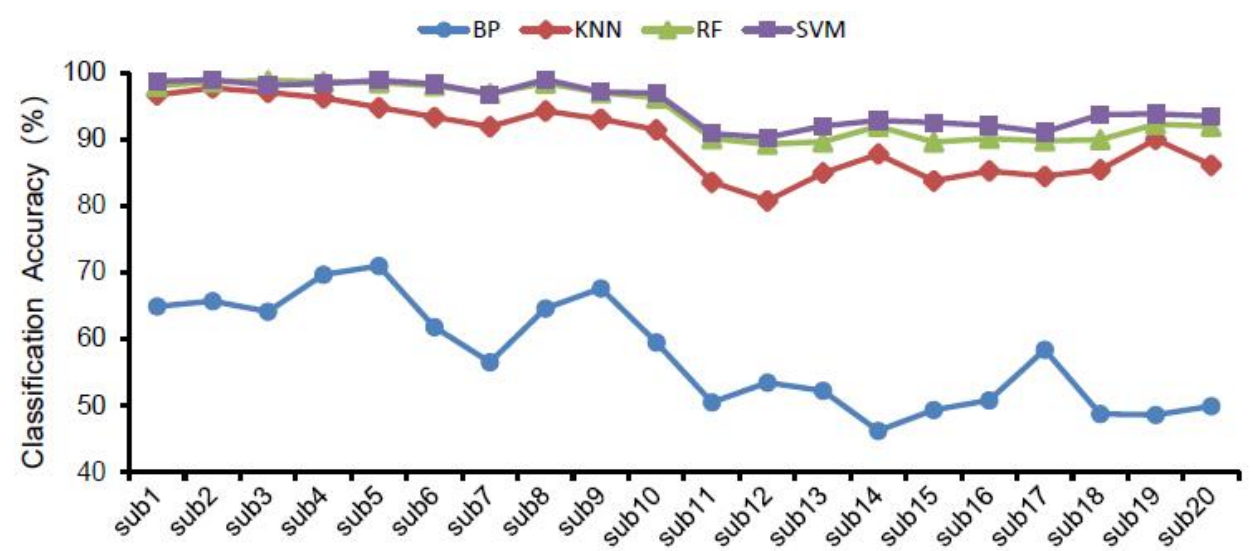

Figure 5. The highest classification accuracy of different classifiers in all bands in 20 subjects.

Specifically, in all wave bands of delta, theta, alpha, beta, and gamma and EEG features including ES, PSD and DE, the SVM classifier had the highest classification accuracy, followed by the RF classifier. In the total band of EEG features such as MAS and PSD, the RF classifier had a slightly higher classification accuracy than the SVM classifier, then the accuracy of the SVM classifier and the RF classifier was significantly higher than the KNN classifier and the BP classifier.

Among the delta, theta, alpha, and beta wave bands of EEG features including RASM and DCAU, the RF classifier had the best classification effect, followed by the SVM classifier. Only in the gamma band, the SVM classifier had a higher classification accuracy than the $\mathrm{RF}$ classifier, the KNN classifier, and the BP classifier.

In the delta and total waves of EEG features DASM, the classification accuracy of the RF classifier was higher than that of the SVM classifier, while in the theta, alpha, beta, and gamma bands, the classification accuracy of the SVM classifier was higher than that of the KNN classifier and the BP classifier.

Using the different classifiers, the trend of landscape recognition accuracy was SVM and $\mathrm{RF}>\mathrm{KNN}>\mathrm{BP}$, while similar trends were confirmed using both the goodness of fit and sensitivity (Table S1).

\subsection{The Classification Effect of Different EEG Features}

In general, for the same brain waves and the same classifier (Table 2 and Figure 6), the classification accuracy of frequency domain features including MAS, PSD and DE was higher than that of spatial domain features including DASM, RASM and DCAU indicators among the six EEG features selected. Furthermore, Figure 7 shows the maximum classification accuracy of different EEG features in 20 subjects in different waves, and also demonstrates that the classification accuracy of frequency domain features is higher than that of spatial domain features. 


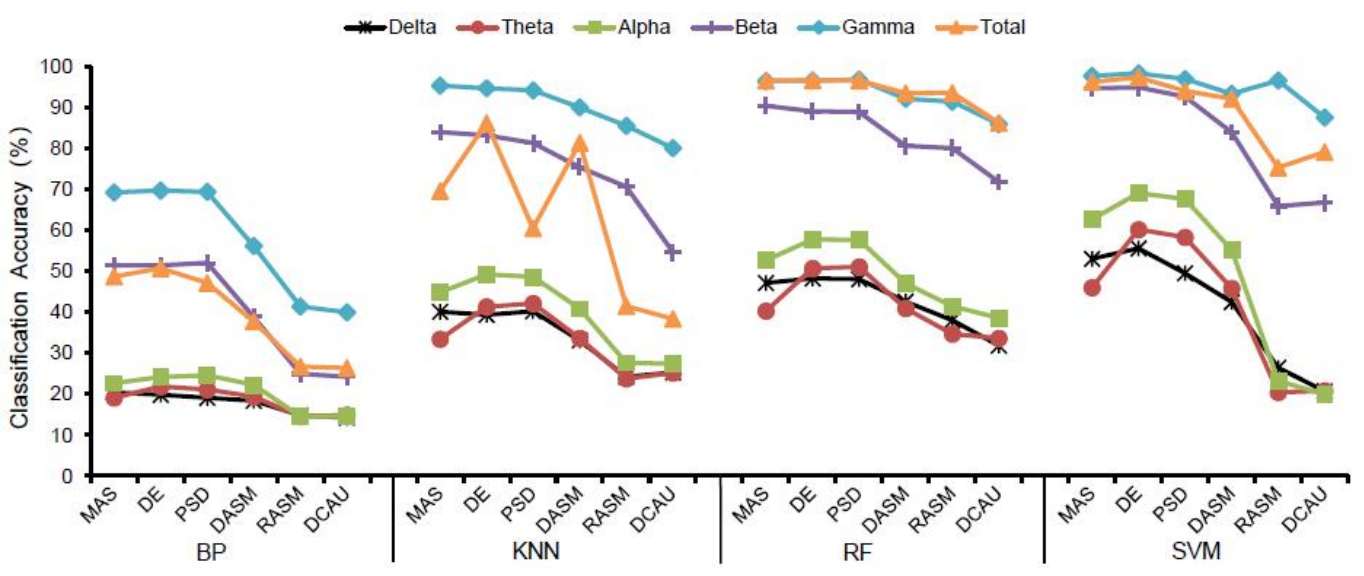

Figure 6. Classification accuracy of different EEG features.

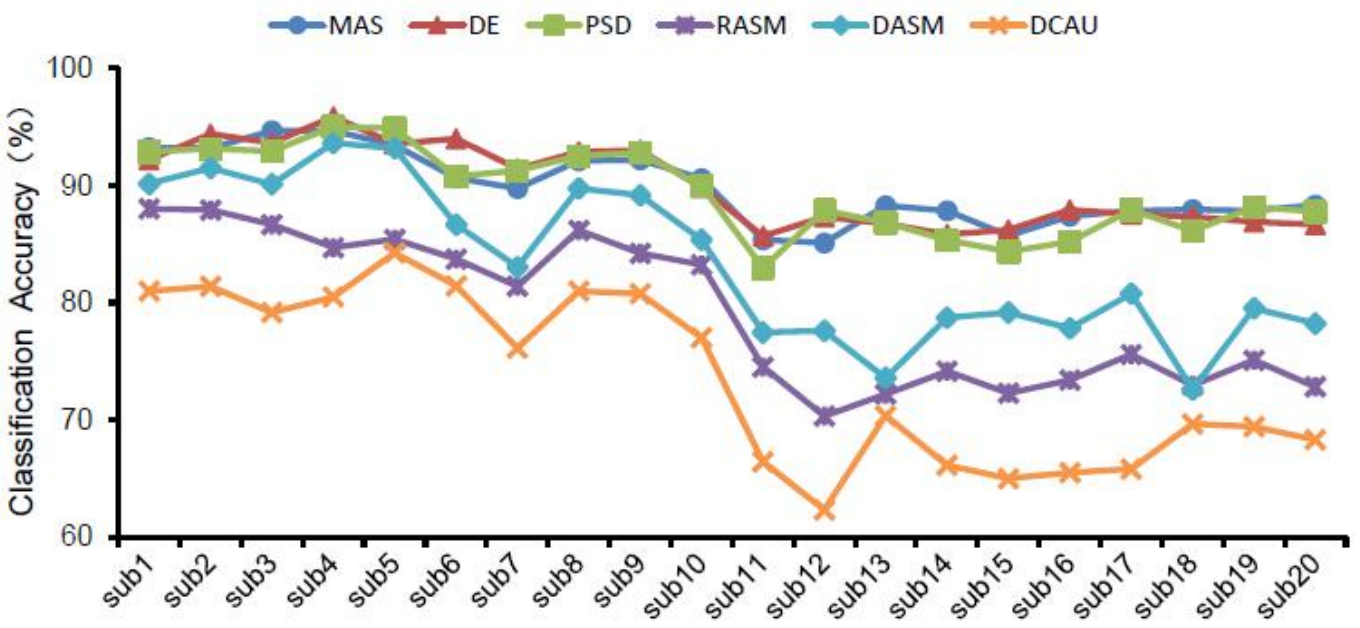

Figure 7. The highest classification accuracy of different EEG features in all bands in 20 subjects.

For the BP classifier, the DE feature had the highest classification accuracy in the delta, theta, gamma, and total wave bands, then PSD was the highest in the alpha band and MAS was the highest in the beta band.

For the KNN classifier, the highest classification accuracy was the PSD feature in the delta and theta wave bands, the DE feature had the highest accuracy in the alpha and total wave bands, and the MAS feature had the highest accuracy in the beta and gamma bands.

For the RF classifier, the classification accuracy of the PSD feature was the highest in the delta, theta, alpha and gamma wave bands, the classification accuracy of the MAS feature was the highest in the beta band, and the accuracy of the DE feature was the highest in the total band.

\subsection{The Classification Effect of Different Brainwave Bands}

With the same selection of classifier and EEG features (Figures 4 and 6), the classification accuracy was highest in the gamma, beta and total bands, and was relatively lower in the delta, theta and alpha bands. Therefore, the recognition of landscape perception by high-frequency waves was more effective than that of low-frequency waves. In all wave bands, the mean classification accuracy of all subjects was $98.24 \%$ in the gamma band, $97.29 \%$ in the total band, and $94.83 \%$ in the beta band. The classification accuracy of the delta band, theta band and alpha band was $55.56 \%, 60.17 \%$ and $69.04 \%$, respectively. This indicates that the classification accuracy of high-frequency waves was higher than that of low-frequency waves in landscape perception recognition and classification. The difference in classification accuracy of different subjects may be related to the subjects' landscape preference (Figures 5 and 7). 
In the BP, KNN and SVM classifiers, the classification accuracy of the gamma band and beta band was higher than that of the total band based on EEG features such as MAS and PSD. The classification accuracy of the gamma band was higher than that of the total band based on EEG features including DE, DASM, RASM, and DCAU. In the RF classifier, there was little difference between the classification accuracy of the gamma band and the total band.

\section{Discussion}

The power of EEG signals in the frequency domain is one of the most commonly used EEG features for emotion analysis. Generally, when the subject is in a calm state, the high amplitude signals in the low frequency range will be more obvious, while in a stimulus state, the high amplitude signals in the high frequency range will be more obvious [28,29]. EEG features (e.g., frequency domain features, features, and spatial domain features) in an EEG signal represent the activities in each brain region. At present, research on recognition and classification based on EEG technology and machine learning have achieved good results.

This study showed that the SVM classifier has the highest classification accuracy in this study. This has been well established in many application fields of EEG technology. In emotion recognition analysis, the frequency domain features that power the spectral density (PSD) of the EEG signals and the asymmetry indexes of the 12 electrode pairs were used to classify discrete emotional states (disgust, happiness, neutrality, sadness, and tenseness) induced by watching a video. A previous study obtained results on the classification accuracy of within-stimulus and found 93.3\% accuracy in the PSD feature, and $85.4 \%$ accuracy in asymmetry features [30]. Shahabi et al. distinguished emotional states (happy, neutral, and melancholy) based on EEG features, and concluded that the classification accuracy between happy and neutral was $93.7 \%$, while the classification accuracy between happy and melancholy was $80.4 \%$ using the SVM classifier [32]. Rasheed et al. classified the EEG signals as red, green, and blue colors and successfully classified the three visual conditions having accuracies of $84 \%, 89 \%$ and $98 \%$ with linear, polynomial, and radial basis function kernels, respectively, within all the groups of data among all the subjects [36]. The extracted PSD features were classified using two-level SVM classifiers in corresponding object-shape classes (including cone, cube, cylinder, sphere, prism, hemisphere, pyramid (square base), hexagonal base cylinder, lock, and mouse) for the three experimental phases. The recognition accuracy was $88.34 \%$ for pure touch and $81.1 \%$ for pure vision, the recognition accuracy of the mixture of tactile and auditory was $82.2 \%$, and the average classification accuracy of the three target recognition modes was 83.89\% [34]. Based on the EEG of prefrontal brain area, SVM was used as a classifier to detect driver fatigue, and the accuracy was $85 \%$ [57]. The recognition of three human stress levels was characterized based on the relative difference between beta and alpha in EEG signals using SVM as classifier, with a recognition accuracy of 75\% [58].

The results show that DE features had the highest classification accuracy in all waves including delta, theta, alpha, beta and gamma and total waves for the SVM classifier. Therefore, DE is an important indicator of landscape perception classification based on EEG technology. Similarly, in the experiment on emotion recognition, using DE as a feature proxy achieves higher recognition accuracy than other features. Zheng et.al used differential entropy, which is a measure of the amount of information included in EEG signals, as the input of deep belief networks, they also used SVM and KNN for the classification of emotional states (positive, neutral, and negative). The classification accuracy was respectively $86.08 \%, 83.99 \%$, and $72.60 \%$ [33]. Differential entropy was also employed to distinguish the positive, neutral, and negative emotional states induced by videos, where the CNN received input as the classifier. The classification accuracy was $83.8 \%$ [31].

This study also showed that the classification accuracy of high-frequency waves was higher than that of low-frequency waves in landscape perception recognition and classification. Moreover, there was similar research suggesting that the gamma band (roughly 30-100 Hz) was suitable for EEG-based emotion classification [59]. The similarity 
with the classification of people's emotions shows that different landscapes do cause subtle changes in people's emotions. At the same time, it also proves that it is feasible to identify people's perception of different landscapes through multi-dimensional EEG features [60]. This result is of great significance for evaluating the impact of landscapes on people, which also has important reference value for the quantification of ecosystem cultural services [44,61].

When the total band of 145 dimensions was used for landscape perception recognition, it did not show the highest accuracy, as it was even lower than the accuracy of landscape perception recognition in the beta band and gamma band. Therefore, more dimensions were not always better $[59,62]$. In this study, the highest classification accuracy of a landscape video was $98.24 \%$ in the gamma band using the SVM classifier. Rus et al. also confirmed that the EEG features of the gamma band are more suitable for object recognition. The classification accuracy of three different classifiers (SVM, KNN and ANN) was 89.5\%, 89.5\% and $83 \%$, respectively [35], which is consistent with the conclusion that the classification accuracy of the gamma band is the highest of all bands in this study. There are relatively few studies on the recognition of landscape types based on EEG technology. Although Lam et al. implemented the single-layer neural network to recognize and classify landscape and animal pictures based on EEG data, the average accuracy was $91.15 \%$, in which the average recognition accuracy of landscape pictures was $89.69 \%$ and that of animal pictures was $92.34 \%$ [37]. However, Lam's research only identified landscape pictures and animal pictures, and did not further identify and classify landscape type pictures.

Compared with the traditional recognition research based on the attribute characteristics of the landscape, this research identifies and classifies landscape perception based on the characteristics of UAV aerial video and EEG features, which provides a certain reference value for the objective quantification of landscape perception evaluation in the future. At the same time, it also provides new ideas and methods for landscape evaluation research, which is worthy of more comprehensive and in-depth research. Future research should focus on the differences in individual perception of landscape physiology, including an increase in sample size and age range and the change of working conditions, as well as further differences in gender, education level, residence, property income and other social attributes. Further research also needs to expand the scope of landscape types. At the same time, more experimental case studies are also necessary. It is very necessary to confirm the conclusions of this study in more complex situations and make them more robust. This study also provides a reference for cultural services of ecosystem services. Compared with the traditional landscape perception evaluation of landscape ecosystems based on questionnaire surveys, more objective multi-dimensional EEG features may be used as a reference in the future.

With the application of brain computer interface technology becoming more and more developed and market-oriented, it is believed that there will be many successful case studies in landscape evaluation and tourism evaluation in the future. There is a need for further research on the relationship between brain activity and landscapes with different characteristics since it is still in the exploratory stage, and there are few relevant studies and generally accepted theoretical basis to explain it. Therefore, it is still a great challenge to verify these relationships with more extensive experimental cases.

\section{Conclusions}

This study identifies and classifies landscape types based on multi-channel and multidimensional EEG features, explores algorithms suitable for landscape perception recognition and classification, preliminarily defines the most suitable frequency band for landscape perception recognition and classification, and compares EEG features with better effects of landscape perception knowledge. It provides a certain reference for landscape perception evaluation in the future.

The results confirmed the following points. For all EEG features, the SVM and RF classifiers have the highest classification accuracy. When the bands are the same and the 
classifiers are the same, the classification accuracy of frequency domain features, MAS, PSD and DE are relatively higher than those of spatial domain features DASM, RASM and DCAU. The DE feature is the most effective among all of the classifiers. For the same classifier and the same EEG feature, the classification accuracy of the beta band and the gamma band is the highest, while the classification accuracy of the delta, theta and alpha bands is relatively lower. Therefore, the high frequency signals are more effective in landscape perception recognition.

Supplementary Materials: The following are available online at https:/ / www.mdpi.com/article/ 10.3390/ijerph19020629/s1, Table S1: The goodness of fit, specificity, and sensitivity of different classifiers in different landscape types.

Author Contributions: Study design, Y.W., M.X.; data collection, Y.W., S.W.; project design, data interpretation, and writing-original draft, Y.W., S.W.; supervision, writing-review and editing, project administration, and funding acquisition, M.X. All authors have read and agreed to the published version of the manuscript.

Funding: This research was funded by the National Key R\&D Program of China Climate Change Impact and Adaptation in Major Countries along the Belt and Road (2018YFA0606500).

Institutional Review Board Statement: The study was conducted according to the guidelines of the Declaration of Helsinki and approved by the Institutional Review Board at Biomedical Research Ethics Sub-Committee of Henan University (protocol code: HUSOM2021-166; date of approval: 15 March 2020).

Informed Consent Statement: Informed consent was obtained from all subjects involved in the study.

Data Availability Statement: The data presented in this study are available in insert article and Supplementary Materials.

Acknowledgments: The authors would like to thank anonymous reviewers for their helpful and stimulating comments and suggestions as well as 20 enthusiastic participants.

Conflicts of Interest: The authors declare no conflict of interest.

\section{References}

1. Blankson, E.J.; Green, B.H. Use of landscape classification as an essential prerequisite to landscape evaluation. Landsc. Urban Plan. 1991, 21, 149-162. [CrossRef]

2. $\quad$ Green, B. Countruside Conservation; Allen and Linwin: London, UK, 1985.

3. Ciglic, R.; Strumbelj, E.; Cesnovar, R.; Hrvatin, M.; Perko, D. Evaluating existing manually constructed natural landscape classification with a machine learning-based approach. J. Spat. Inf. Sci. 2019, 18, 31-56. [CrossRef]

4. Fňukalová, E.; Romportl, D. A typology of natural landscapes of Central Europe. AUC Geogr. 2014, 49, 57-63. [CrossRef]

5. Liang, F.; Liu, L. Research progress and development trend of landscape classification. J. Appl. Ecol. 2011, 22, $1632-1638$.

6. Franklin, S.E.; Ahmed, O.S. Deciduous tree species classification using object-based analysis and machine learning with unmanned aerial vehicle multispectral data. Int. J. Remote Sens. 2018, 39, 5236-5245. [CrossRef]

7. Liu, J.; Feng, Q.; Gong, J.; Zhou, J.; Liang, J.; Li, Y. Winter wheat mapping using a random forest classifier combined with multi-temporal and multi-sensor data. Int. J. Digit. Earth 2018, 11, 783-802. [CrossRef]

8. Matikainen, L.; Karila, K.; Hyyppa, J.; Litkey, P.; Puttonen, E.; Ahokas, E. Object-based analysis of multispectral airborne laser scanner data for land cover classification and map updating. ISPRS. J. Photogramm. 2017, 128, 298-313. [CrossRef]

9. Daniel, T.C.; Boster, R.S. Measuring Landscape Esthetics: The Scenic Beauty Estimation Metho; Department of Agriculture, Forest Service, Rocky Mountain Forest and Range Experiment Station: Washington, DC, USA, 1976.

10. Daniel, T.C.; Vining, J. Methodological issues in the assessment of landscape quality. In Behavior and the Natural Environment; Springer: Boston, MA, USA, 1983; pp. 39-84. [CrossRef]

11. Smrekar, A.; Horvat, K.P.; Erhartič, B. The beauty of landforms. Acta Geogr. Slov. 2016, 56, 321-335. [CrossRef]

12. Tenerelli, P.; Püffel, C.; Luque, S. Spatial assessment of aesthetic services in a complex mountain region: Combining visual landscape properties with crowdsourced geographic information. Landsc. Ecol. 2017, 32, 1097-1115. [CrossRef]

13. Kolbovsky, E.Y.; Medovikova, U. Assessment of the aesthetic landscape properties for managing areas of outstanding natural beauty and historical significance. Reg. Res. Russ. 2017, 7, 81-88. [CrossRef]

14. Root, E.; Silbernagel, K.; Litt, J. Unpacking healthy landscapes: Empirical assessment of neighborhood aesthetic ratings in an urban setting. Landsc. Urban Plan. 2017, 168, 38-47. [CrossRef] 
15. Marshall, N.; Marshall, P.; Curnock, M.; Pert, P.; Smith, A.; Visperas, B. Identifying indicators of aesthetics in the Great Barrier Reef for the purposes of management. PLoS ONE 2019, 14, e0210196. [CrossRef] [PubMed]

16. Meitner, M.J. Scenic beauty of river views in the Grand Canyon: Relating perceptual judgments to locations. Landsc. Urban Plan. 2004, 68, 3-13. [CrossRef]

17. Carlson, A. Nature and Landscape: An Introduction to Environmental Aesthetics; Columbia University Press: New York, NY, USA, 2009.

18. Stuart-Smith, S. The Well-Gardened Mind: The Restorative Power of Nature; William Collins: London, UK, 2020.

19. Appleton, J. The Experience of Landscape; Wiley Chichester: Chichester, UK, 1996.

20. Kellert, S.R.; Wilson, E.O. The Biophilia Hypothesis; Island Press: Washington, DC, USA, 1993.

21. Coss, R.G.; Moore, M. Precocious knowledge of trees as antipredator refuge in preschool children: An examination of aesthetics, attributive judgments, and relic sexual dinichism. Ecol. Psychol. 2002, 14, 181-222. [CrossRef]

22. Bauske, E.M.; Waltz, C. Influence of turfgrass on human aesthetics and psychology: A review. Acta Hortic. 2013, 999, 37-41. [CrossRef]

23. Schafer, P.J.; Serman, M.; Arnold, M.; Corona-Strauss, F.I.; Strauss, D.J.; Seidler-Fallbohmer, B.; Seidler, H. Evaluation of an objective listening effort measure in a selective, multi-speaker listening task using different hearing aid settings. In Proceedings of the 2015 37th Annual International Conference of the IEEE Engineering in Medicine and Biology Society (EMBC), Milan, Italy, 25-29 August 2015; pp. 4647-4650. [CrossRef]

24. Keshavarz, B.; Campos, J.L.; Berti, S. Vection lies in the brain of the beholder: EEG parameters as an objective measurement of vection. Front. Psychol. 2015, 6, 1581. [CrossRef]

25. Norwood, M.F.; Lakhani, A.; Maujean, A.; Zeeman, H.; Kendall, E. Brain activity, underlying mood and the environment: A systematic review. J. Environ. Psychol. 2019, 65, 101321. [CrossRef]

26. Teplan, M. Fundamentals of EEG measurement. Meas. Sci. Rev. 2002, 2, 1-11.

27. Whalen, P.J.; Rauch, S.L.; Etcoff, N.L.; McInerney, S.C.; Lee, M.B.; Jenike, M.A. Masked Presentations of Emotional Facial Expressions Modulate Amygdala Activity without Explicit Knowledge. J. Neurosci. 1998, 18, 411-418. [CrossRef] [PubMed]

28. Bear, M.; Connors, B.; Paradiso, M. Neuroscience: Exploring the Brain; Jones \& Bartlett Learning: Burlington, VT, Canada, 2015.

29. Moon, S.E.; Chen, C.J.; Hsieh, C.J.; Wang, J.L.; Lee, J.S. Emotional EEG classification using connectivity features and convolutional neural networks. Neural Netw. 2020, 132, 96-107. [CrossRef] [PubMed]

30. Liu, S.; Tong, J.; Meng, J.; Yang, J.; Zhao, X.; He, F.; Qi, H.; Ming, D. Study on an effective cross-stimulus emotion recognition model using EEGs based on feature selection and support vector machine. Int. J. Mach. Learn. Cybern. 2018, 9, 721-726. [CrossRef]

31. Li, J.; Zhang, Z.; He, H. Hierarchical convolutional neural networks for EEG-based emotion recognition. Cogn. Comput. 2018, 10, 368-380. [CrossRef]

32. Shahabi, H.; Moghimi, S. Toward automatic detection of brain responses to emotional music through analysis of EEG effective connectivity. Comput. Hum. Behav. 2016, 58, 231-239. [CrossRef]

33. Zheng, W.L.; Lu, B.L. Investigating critical frequency bands and channels for EEG-based emotion recognition with deep neural networks. IEEE Trans. Auton. Ment. Dev. 2015, 7, 162-175. [CrossRef]

34. Khasnobish, A.; Konar, A.; Tibarewala, D.; Bhattacharyya, S.; Janarthanan, R. Object shape recognition from EEG signals during tactile and visual exploration. In Proceedings of the International Conference on Pattern Recognition and Machine Intelligence, Kolkata, India, 10-14 December 2013; pp. 459-464. [CrossRef]

35. Rus, I.D.; Marc, P.; Dinsoreanu, M.; Potolea, R.; Muresan, R.C. Classification of EEG signals in an object recognition task. In Proceedings of the 2017 13th IEEE International Conference on Intelligent Computer Communication and Processing (ICCP), Cluj-Napoca, Romania, 7-9 September 2017; pp. 391-395. [CrossRef]

36. Rasheed, S.; Marini, D. Classification of EEG signals produced by RGB colour stimuli. J. Biomed. Eng. Med. 2015, 2, 56. [CrossRef]

37. Lam, Q.C.; Nguyen, L.A.T.; Nguyen, H.K. EEG Signal Identification Using Single-Layer Neural Network. Int. J. Cybern. Inform. 2016, 5, 1-10. [CrossRef]

38. Chang, C.Y.; Hammitt, W.E.; Chen, P.K.; Machnik, L.; Su, W.C. Psychophysiological responses and restorative values of natural environments in Taiwan. Landscape. Urban. Plan. 2008, 85, 79-84. [CrossRef]

39. Roe, J.J.; Aspinall, P.A.; Mavros, P.; Coyne, R. Engaging the brain: The impact of natural versus urban scenes using novel EEG methods in an experimental setting. Environ. Sci. 2013, 1, 93-104. [CrossRef]

40. Tilley, S.; Neale, C.; Patuano, A.; Cinderby, S. Older People's Experiences of Mobility and Mood in an Urban Environment: A Mixed Methods Approach Using Electroencephalography (EEG) and Interviews. Int. J. Environ. Res. Public Health 2017, 14, 151. [CrossRef]

41. Ulrich, R.S. Natural versus urban scenes: Some psychophysiological effects. Environ. Behav. 1981, 13, 523-556. [CrossRef]

42. Chiang, Y.C.; Li, D.Y.; Jane, H.A. Wild or tended nature? The effects of landscape location and vegetation density on physiological and psychological responses. Landsc. Urban Plan. 2017, 167, 72-83. [CrossRef]

43. Qin, J.; Zhou, X.; Sun, C.; Leng, H.; Lian, Z. Influence of green spaces on environmental satisfaction and physiological status of urban residents. Urban For. Urban Green. 2013, 12, 490-497. [CrossRef]

44. Sacchelli, S.; Grilli, G.; Capecchi, I.; Bambi, L.; Barbierato, E.; Borghini, T. Neuroscience Application for the Analysis of Cultural Ecosystem Services Related to Stress Relief in Forest. Forests 2020, 11, 190. [CrossRef]

45. Hu, W.; Cao, J.; Lai, X.; Liu, J. Mean amplitude spectrum based epileptic state classification for seizure prediction using convolutional neural networks. J. Ambient. Intell. Human. Comput. 2019. [CrossRef] 
46. Duan, R.N.; Zhu, J.Y.; Lu, B.L. Differential entropy feature for EEG-based emotion classification. In Proceedings of the 2013 6th International IEEE/EMBS Conference on Neural Engineering (NER), San Diego, CA, USA, 6-8 November 2013; pp. 81-84. [CrossRef]

47. Shi, L.C.; Jiao, Y.Y.; Lu, B.L. Differential entropy feature for EEG-based vigilance estimation. In Proceedings of the 2013 35th Annual International Conference of the IEEE Engineering in Medicine and Biology Society (EMBC), Osaka, Japan, 3-7 July 2013; pp. 6627-6630. [CrossRef]

48. Duan, R.N.; Wang, X.W.; Lu, B.L. EEG-Based Emotion Recognition in Listening Music by Using Support Vector Machine and Linear Dynamic System. In Neural Information Processing; ICONIP 2012. Lecture Notes in Computer Science; Huang, T., Zeng, Z., Li, C., Leung, C.S., Eds.; Springer: Berlin/Heidelberg, Germany, 2012; Volume 7666. [CrossRef]

49. Pivik, R.T.; Broughton, R.J.; Coppola, R.; Davidson, R.J.; Fox, N.; Nuwer, M.R. Guidelines for the recording and quantitative analysis of electroencephalographic activity in research contexts. Psychophysiology 1993, 30, 547-558. [CrossRef] [PubMed]

50. Keil, A.; Debener, S.; Gratton, G.; Junghöfer, M.; Kappenman, E.S.; Luck, S.J.; Luu, P.; Miller, G.A.; Yee, C.M. Committee report: Publication guidelines and recommendations for studies using electroencephalography and magnetoencephalography. Psychophysiology 2014, 51, 1-21. [CrossRef] [PubMed]

51. Fridlund, A.J.; Cacioppo, J.T. Guidelines for human electromyographic research. Psychophysiology 1986, 23, 567-589. [CrossRef]

52. Chaumon, M.; Bishop, D.V.; Busch, N.A. A practical guide to the selection of independent components of the electroencephalogram for artifact correction. J. Neurosci. Methods 2015, 250, 47-63. [CrossRef]

53. Gabard-Durnam, L.J.; Wilkinson, C.; Kapur, K.; Tager-Flusberg, H.; Levin, A.R.; Nelson, C.A. Longitudinal EEG power in the first postnatal year differentiates autism outcomes. Nat. Commun. 2019, 10,1-12. [CrossRef]

54. Qiao, S.; Sedillo, J.I.; Brown, K.A.; Ferrentino, B.; Pesaran, B. A Causal Network Analysis of Neuromodulation in the Mood Processing Network. Neuron 2020, 107, 972-985. [CrossRef]

55. Conneau, A.C.; Essid, S. Assessment of new spectral features for eeg-based emotion recognition. In Proceedings of the 2014 IEEE International Conference on Acoustics, Speech and Signal Processing (ICASSP), Florence, Italy, 4-9 May 2014. [CrossRef]

56. Chang, C.C.; Lin, C.J. LIBSVM: A library for support vector machines. ACM Trans. Intell. Syst. Technol. 2011, 2, 1-27. [CrossRef]

57. $\mathrm{Mu}, \mathrm{Z} . ; \mathrm{Hu}, \mathrm{J} . ;$ Yin, J. Driving fatigue detecting based on EEG signals of forehead area. Int. J. Pattern. Recogn. 2017, 31, 1750011. [CrossRef]

58. Jun, G.; Smitha, K.G. EEG based stress level identification. In Proceedings of the 2016 IEEE International Conference on Systems, Man, and Cybernetics (SMC), Budapest, Hungary, 9-12 October 2016; pp. 3270-3274. [CrossRef]

59. Li, M.; Lu, B.L. Emotion classification based on gamma-band EEG. In Proceedings of the 2009 Annual International Conference of the IEEE Engineering in medicine and biology society, Minneapolis, MN, USA, 3-6 September 2009; pp. 1223-1226. [CrossRef]

60. Wang, Y.; Xu, M. Electroencephalogram Application for the Analysis of Stress Relief in the Seasonal Landscape. Int. J. Environ. Res. Public Health 2021, 18, 8522. [CrossRef] [PubMed]

61. Wang, Y.; Wang, S.; Xu, M. The Function of Color and Structure Based on EEG Features in Landscape Recognition. Int. J. Environ. Res. Public Health 2021, 18, 4866. [CrossRef] [PubMed]

62. Zhu, J.Y.; Zheng, W.L.; Peng, Y.; Duan, R.N.; Lu, B.L. EEG-based emotion recognition using discriminative graph regularized extreme learning machine. In Proceedings of the 2014 International Joint Conference on Neural Networks (IJCNN), Beijing, China, 6-11 July 2014; pp. 525-532. [CrossRef] 